\title{
Factor C-comunidad, factor dinamizador en las organizaciones solidarias
}

\author{
Luz Miriam Valencia Velásquez ${ }^{\star}$, Luis Alberto Gallego Moná ${ }^{*}$
}

* Especialista en Docencia Universitaria. Magíster (c) en Gestión de Organizaciones. Profesora de tiempo completo, Universidad Cooperativa de Colombia, sede Medellín.

Correo electrónico: luz.valencia@campusucc.edu.co,

** Administrador de Empresas. Asistente de investigación de la Facultad de Ciencias Económicas, Administrativas y Afines, Universidad Cooperativa de Colombia, sede Medellín.

Correo electrónico: luis.gallego@campusucc.edu.co

Recibido: 10 de junio del 2013

Aprobado: 30 de octubre del 2013

Cómo citar este artículo: Valencia Velásquez, L.M. y Gallego Moná, L.A. (2013). Factor C-comunidad, factor dinamizador en las organizaciones solidarias. Cooperativismo \& Desarrollo, 21(103), 69-80.

\begin{abstract}
Resumen
El presente artículo es resultado de la investigación desarrollada en las asociaciones y cooperativas de pequeños y medianos productores agropecuarios, vinculadas a la economía solidaria de las zonas de Sinifaná y San Juan, en la región del suroeste antioqueño. Se propone una reflexión sobre el Factor C-comunidad, como el factor que integra la propuesta asociativa en estas organizaciones que trabajan con el objetivo de satisfacer las necesidades, las aspiraciones y los deseos de las personas, las familias y las comunidades en las que operan. La metodología utilizada en la investigación parte del enfoque cualitativo de tipo etnográfico, en la cual se utilizaron técnicas tanto cualitativas como cuantitativas, permitiendo comprender la dinámica de las organizaciones. Los resultados más relevantes llevaron a identificar el Factor C-comunidad como un objetivo socioeconómico efectivamente integrador que, operando en combinación con otros factores productivos, se convierte en la fuerza que une voluntades y conciencias hacia el mejoramiento de la calidad de vida de sus integrantes, la rentabilidad de la organización y su entorno.
\end{abstract}

Palabras clave: asociaciones, factor comunidad, productores agropecuarios, solidaridad.

\section{The C-Factor: Community as a Dynamizing Force in Solidarity Organizations}

\begin{abstract}
This article is the result of research conducted in associations and cooperatives of small and medium-size agricultural producers linked to the solidarity economy in the Sinifaná and San Juan areas of southeast Antioquia, Colombia. The article examines the community factor, or C-factor, as a factor that integrates the collective goals of these organizations, which seek to satisfy the needs, aspirations and wishes of the people, families and communities where they operate. While an ethnographic qualitative approach was used as the starting point for the methodology in this research, it incorporates both qualitative and quantitative techniques, allowing an understanding of the dynamics of these organizations. The most relevant results identified the C-Factor as a socio-economic integrating objective which, in combination with other productive factors, becomes a force that unites desires and builds awareness to improve the quality of life for its participants, as well as the profitability of the organization and its environment.
\end{abstract}

Keywords: associations, community factor, agricultural producers, solidarity.

\section{Fator C-comunidade, fator dinamizador nas organizações solidárias}

\section{Resumo}

O presente artigo é resultado da pesquisa desenvolvida nas associações e cooperativas de pequenos e médios produtores agropecuários, vinculadas à economia solidária das zonas de Sinifaná e San Juan, na região do sudoeste da Antioquia (Colômbia). Propõe-se uma reflexão sobre o Fator C-comunidade, como o fator que integra a proposta associativa nessas organizações que trabalham com o objetivo de satisfazer as necessidades, aspirações e desejo das pessoas, famílias e das comunidades nas quais operam. A metodologia utilizada na pesquisa parte do enfoque qualitativo de tipo etnográfico, na qual se utilizaram técnicas tanto qualitativas quanto quantitativas, o que permitiu compreender a dinâmica das organizações. Os resultados mais relevantes levaram a identificar o Fator C-comunidade como um objetivo socioeconômico efetivamente integrador que, ao operar em combinação com outros fatores produtivos, se converte na força que une vontades e consciências em prol do melhoramento da qualidade de vida de seus integrantes, da rentabilidade da organização e de seu ambiente.

Palavras-chave: associações, fator comunidade, produtores agropecuários, solidariedade. 


\section{Introducción ${ }^{1}$}

La investigación permitió hacer una representación amplia y profunda de los factores económicos que forman parte integral de una organización altamente productiva; asimismo, logró caracterizar las asociaciones y cooperativas de pequeños y medianos productores agropecuarios que han asumido la responsabilidad de la autogestión y han potencializado las capacidades de las personas, para contribuir con la construcción del propio desarrollo y del entorno que recibe su influencia. Dicha caracterización requirió el reconocimiento de los factores gestión, tecnología, trabajo y el Factor Integrador C-comunidad planteado en la Teoría Económica Comprensiva expuesta por el profesor chileno Luis Razeto (Razeto, 1994b).

El Factor C-comunidad es un elemento que las empresas de hoy en día no consideran realmente importante por su desconocimiento y poco interés sobre las nuevas teorías expuestas. La investigación que se realizó en las zonas de Sinifaná y San Juan, en el suroeste antioqueño, sobre el Factor C-comunidad, permitió identificar las características y la concepción de las comunidades en la manera como logran asociarse para alcanzar las metas que se han trazado, pero, sobre todo, y lo más importante, descubrir si realmente el Factor C-comunidad es el factor integrador que permite que las personas se unan para desarrollar una actividad productiva.

Se pudieron identificar las formas de asociatividad y cooperativismo que desarrollan los habitantes de una región; qué conocimiento poseen sobre el cooperativismo en general; y si conocen los pasos y la normatividad que se requiere para crear una asociación o una cooperativa. Todos estos aspectos fueron importantes en la investigación porque permitieron finalmente realizar un análisis global sobre las diversas estructuras

1. El artículo es el resultado de la investigación "Caracterización de las cooperativas y asociaciones de pequeños y medianos productores agropecuarios de las zonas de Sinifaná y San Juan, del suroeste antioqueño, financiada por la Corporación Universitaria Lasallista, la Fundación Universitaria Luis Amigó, la Universidad Cooperativa de Colombia y la Facultad de Salud Pública de la Universidad de Antioquia. Realizada por Sol Bibiana Mora Rendón, del grupo de investigación Delta, clasificado en Colciencias en categoría C; Luz Dolly Lopera García, del grupo de investigación Ecosol, clasificado en Colciencias en categoría B; Luz Mirian Valencia, del grupo de investigación gies, clasificado en Colciencias como visible. En calidad de coinvestigadores participaron Francisco Aguirre Echevarría y Olga Lucía Arboleda, de la Facultad de Ciencias Administrativas, Económicas y Contables de la Funlam, Medellín, Colombia, y Luis Fernando Ospina, de la Universidad Cooperativa de Colombia, sede Medellín. asociativas ubicadas en las regiones y comunidades objeto de la investigación.

También se logró establecer un nuevo conocimiento sobre el saber y el concepto que tienen las organizaciones de esta región sobre el Factor C-comunidad; qué entienden por economía solidaria; si ellos consideran importante la implementación y ejecución de la solidaridad en la realización de sus labores diarias. Es claro que la mayor parte de la sociedad está absorbida por una economía capitalista, pues allí se encuentra inmersa la mayor parte de las personas y las empresas; sin embargo, se debe reconocer y resaltar que la economía capitalista es sólo una porción de la economía como tal y no un todo global, puesto que la economía solidaria también forma parte de dicho entorno, y cada día crece con más fuerza por la diversidad de beneficios y servicios que obtienen sus asociados cuando se realiza una excelente función en torno a un objetivo común como es el bienestar de todos los integrantes de dicho modelo.

Hoy en día, la economía capitalista no es tan fuerte como años atrás, es evidente la crisis en la que han caído un gran número de empresas. Esto genera que se presenten consecuencias que afectan la calidad de vida de la población, luego no se brindan las mismas oportunidades ni posibilidades de empleo que se requerirían en una situación ideal, por tanto las condiciones del mercado han cambiado y es allí donde la economía solidaria cobra mayor importancia, un papel protagónico y relevante para la sociedad, pues las personas acuden a ella como posible solución a los inconvenientes y las dificultades que se les presentan en el mejoramiento de su calidad de vida.

Tabla 1. Subregiones del departamento de Antioquia

\begin{tabular}{|l|l|c|}
\hline \multicolumn{1}{|c|}{ Subregión } & \multicolumn{1}{|c|}{ Nombre turístico } & $\begin{array}{c}\text { Número de } \\
\text { municipios }\end{array}$ \\
\hline Valle de Aburrá & Luces, Paisajes y Flores & 10 \\
\hline Magdalena Medio & Agua, Bosque, Cemento & 6 \\
\hline Occidente & Sol y Frutas & 18 \\
\hline Oriente & Ruta Verde & 23 \\
\hline Norte & Ruta de la Leche & 17 \\
\hline Urabá & Mar de Alegría, Riqueza. Placer & 11 \\
\hline Suroeste & Tradición Café, Carbón y & 24 \\
\hline Nordeste & Arriería & 10 \\
\hline Bajo Cauca & Tren de Oro y Caña & 6 \\
\hline \multicolumn{2}{r|}{ Ruta de Oro, Ganado y Pesca } & 125 \\
\cline { 2 - 3 } & &
\end{tabular}

Fuente: Gobernación de Antioquia, 2004 


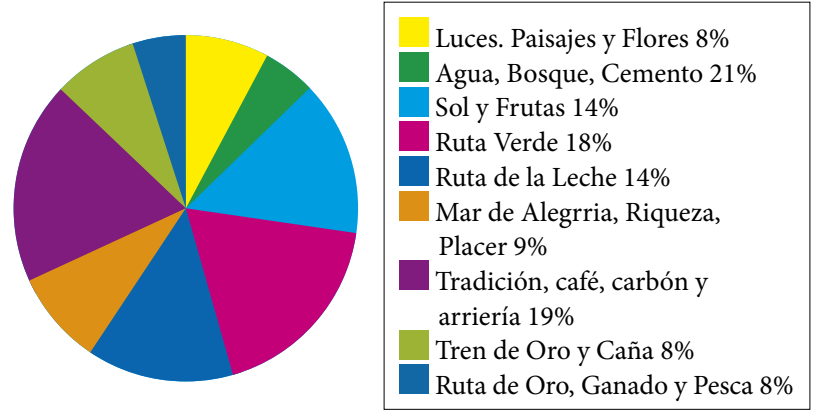

Figura 1. Subregiones del departamento de Antioquia Fuente: Gobernación de Antioquia, 2004

Antioquia es un departamento de Colombia localizado al noroeste del país. Ocupa un territorio de 63.612 kilómetros cuadrados y está habitado por 6.299 .990 personas en un total de 125 municipios representados en nueve subregiones (tabla 1). Estas subregiones están identificadas por características, en su mayoría de índole turística, que las hace altamente atractivas y a su vez ser definidas productivamente dentro de la región (figura 1). Más de la mitad de sus habitantes se encuentran ubicados en el área metropolitana del valle de Aburrá. La economía de Antioquia genera el 13\% del PIB del país, ubicándose en segundo lugar después de Bogotá, su capital.

La investigación se realizó en el suroeste antioqueño por su importancia a nivel departamental y nacional como una subregión con economía diversificada, infraestructura competitiva y sostenible en agroindustria, turismo y minería, con desarrollo humano integral, equitativo y justo, en continuo respeto de los derechos humanos y con proyección nacional e internacional (Antioquia, Gobernación de Antioquia, 2004). La región suroeste está compuesta por 4 zonas (tabla 2), de las cuales se tomaron para el estudio las de Sinifaná y San Juan. Subzonas con características muy similares en número de municipios que las conforman y en actividades productivas de participación (figura 2 ).

Tabla 2. Zonas de la subregión suroeste del departamento de Antioquia

\begin{tabular}{|l|c|}
\hline \multicolumn{1}{|c|}{ Zonas } & Número de municipios \\
\hline Sinifaná & 5 \\
\hline San Juan & 5 \\
\hline Cartama & 9 \\
\hline Penderisco & 5 \\
\hline
\end{tabular}

Fuente: Gobernación de Antioquia, 2004

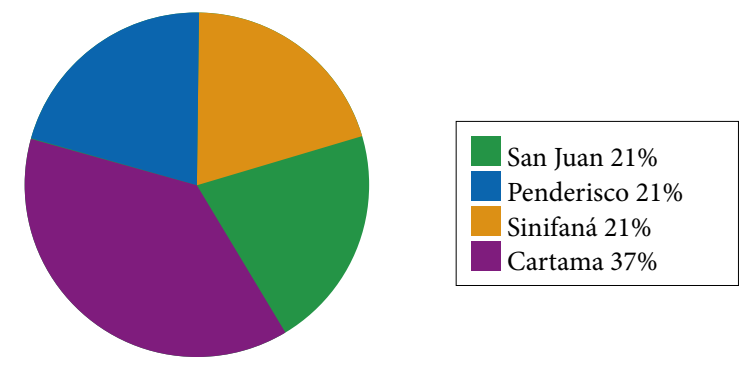

Figura 2. Porcentaje de participacion por numero de municipios en la subregion del suroeste Fuente: Gobernación de Antioquia, 2004

\section{Fundamentación teórica}

El concepto de Factor C-comunidad es de reciente acuñación y no es aún reconocido en las teorías económicas convencionales. Por ello, es conveniente detenernos un momento en la explicación de su contenido, sus orígenes, sus modalidades de presencia en las empresas y en la economía en general, y sus modos de operar y contribuir a la producción. Tal vez una buena forma de iniciar la explicación sea contar que descubrimos este factor haciendo un análisis técnico de numerosas organizaciones económicas populares, surgidas en un contexto de fuerte crisis económica, como respuesta de grupos de personas desplazadas de sus puestos de trabajo y que experimentaron un grave problema de supervivencia (Razeto, 2011a).

Se denomina Factor C-comunidad, porque con esta letra comienzan muchos términos que lo identifican: cooperación, comunidad, colaboración, compañerismo, comunión, compartir, etcétera. Con este factor superaban las limitaciones y carencias de todos y cada uno de los demás factores, inyectándoles un plus de energía social y de eficiencia que explicaba gran parte del producto. Las carencias tecnológicas las superaban compartiendo conocimientos fragmentarios y trabajando en equipo en la búsqueda de soluciones a los problemas técnicos: es impresionante la creatividad personal y colectiva que se genera cuando las personas se unen para enfrentar una dificultad que deben resolver en común.

Las carencias de gestión eran superadas recurriendo a la consulta, la toma de decisiones en común, la participación y la autogestión. Las limitaciones de financiamientos y medios materiales se resolvían con base en la confianza mutua y la fe en el proyecto común, que les hacía entregar su trabajo y sus pequeños aportes de 
dinero y bienes con la seguridad de que serían recompensados con los resultados de su utilización. Sustituían de este modo, con Factor C, los otros factores de producción que poseían muy limitadamente, estableciendo una combinación que daba lugar a pequeñas empresas intensivas en este factor comunitario (Razeto, 2011b).

El Factor C-comunidad es el pilar fundamental por medio del cual las comunidades más necesitadas de la sociedad pueden lograr obtener grandes beneficios y resultados, puesto que unen sus esfuerzos y capacidades con el ánimo y la intención de cumplir una meta u objetivo en particular y trabajar en unión, solidaridad, comunidad y compañerismo como base fundamental de una sociedad.

Desde los comienzos del capitalismo moderno y hasta nuestros días, gran parte de la humanidad ha querido, esperado o perseguido activamente un cambio profundo en los modos de organización y operación de la economía. Muchos aspiran al cambio porque experimentan la marginación, la exclusión y la pobreza, o porque sufren directamente la injusticia de este sistema. Otros buscan el cambio porque los modos de operar, de relacionarse y de organizar los procesos de producción, distribución, consumo y acumulación capitalistas - basados en el afán desmedido del lucro, la competencia desenfrenada, el consumismo, la subordinación del trabajo al capital—, contradicen sus ideales, sus valores, sus principios intelectuales, morales y espirituales (Razeto, 1994b).

El desempleo es una problemática que cada día se presenta con mayor fuerza y esto se debe a una serie de circunstancias que ocurren tanto en el mercado interno como externo; por este motivo, muchas personas buscan oportunidades en las ciudades. Es así como una gran cantidad de comunidades tratan de fortalecer las políticas agroindustriales de sus regiones para que los habitantes no tengan que desplazarse a las ciudades en busca de oportunidades, si no que su región sea de gran capacidad productiva. Es allí donde el Factor Ccomunidad juega un papel fundamental debido a que es la alternativa en la cual se refugian y, por medio de la unión de esfuerzos para el desarrollo, contando con los recursos que tienen disponibles, iniciar así proyectos que les permitan consolidarse en una realidad local.

La sociedad actual, con todo su componente de tecnologías, comunicaciones y economía globalizada, no sólo está sembrando el planeta de fantásticas herramientas, sino que también está transformando los valores que durante siglos han dirigido los pasos de la humanidad. Una de estas transformaciones es el surgimiento del cambio como valor clave para los habitantes del presente. Importado desde el mundo productivo, el paradigma del cambio surge como la necesidad de las empresas de diversificar su oferta para adaptarse a los múltiples mercados, públicos y requerimientos, y para superar la competencia. Esto exige una constante renovación, que va desde los productos que se ofrecen, hasta la propia gestión organizacional.

Así, en un mundo en el que la producción es clave para la construcción de la sociedad y de la identidad de las personas, este nuevo enfoque no tarda en extenderse al resto del conglomerado social, y particularmente al espacio de las relaciones entre personas. A escala humana esta transformación permanente exige que el hombre y la mujer desarrollen al máximo sus capacidades de flexibilidad, tolerancia y adaptación a lo nuevo $y$, al mismo tiempo, desechen todo indicio de inmovilidad y estancamiento (Prieto, 2006).

La escasez de recursos en la economía de hoy es evidente; es claro que día a día son demasiadas las comunidades que se enfrentan a una serie de retos y dificultades, puesto que muchas empresas del sector privado deben cerrar sus puertas por que no cuentan con la cantidad de recursos suficientes para continuar con la operatividad del negocio y, debido a esto, el recurso humano que labora en dichas empresas deben buscar la forma de enfrentar y solucionar estas dificultades, pues tienen un serio compromiso con sus familias para lograr subsistir con las comodidades mínimas; esto se evidenció en las comunidades objeto de la investigación: los habitantes de las veredas laboran en grandes fincas cafeteras o de otro tipo de cultivo, donde por realizar su trabajo devengan una determinada cantidad de dinero, mínima en muchos casos, denominada jornal.

Es muy usual que cuando hay cosecha de productos se presente gran demanda de personal, puesto que para recolectar la totalidad de la cosecha se requiere de suficiente mano de obra; son las mejores épocas del año tanto para los propietarios de las fincas como para el recursos humano de la región, se presenta una bonanza económica importante. Sin embargo, cuando la recolección termina, los propietarios de las fincas ya no requieren de los servicios de estas personas, y por consiguiente son despedidos; allí se presenta nuevamente una escasez de recursos económicos para la población menos favorecida que habita en dicha comunidad.

Los habitantes de la región objeto de estudio viven año tras año con la realidad de tener recursos económicos en gran cantidad sólo en ciertas épocas, pero también que se presentan tiempos de escasez, en los que 
deciden que la mejor propuesta es unir sus esfuerzos e ideas para lograr desarrollar proyectos productivos de su propia iniciativa, alrededor de lo que saben y vienen haciendo, y que sea para el beneficio de toda la población donde convergen.

Es allí donde el Factor C-comunidad juega un papel fundamental para lograr desarrollar y ejecutar satisfactoriamente sus ideales. Los habitantes de la región unen sus recursos económicos, trabajo, experiencias y saberes, y hacen todo lo que esté a su alcance para lograr consolidar todo lo necesario y requerido para iniciar las asociaciones y cooperativas, motivados por las características del Factor C-comunidad, que en la mayoría de las ocasiones no se lograr identificar y percibir como tal, al unir sus esfuerzos y capacidades. Sin duda alguna, este es el factor que se convierte en el pilar para el desarrollo económico y la proyección social de las comunidades.

Formulado el concepto, era fácil darse cuenta de que el Factor C-comunidad no es exclusivo de las unidades económicas populares surgidas en un contexto de crisis, sino que toda empresa lo necesita y utiliza, y que en todas las unidades económicas manifiesta una propia y especial productividad. Su esencia no es otra cosa que la energía social que surge de la unión de conciencias, sentimientos y voluntades tras un objetivo común. En realidad, esta energía es extraordinariamente poderosa y se manifiesta en todo tipo de organizaciones y actividades sociales. La experiencia histórica enseña, en efecto, que muchas veces un ejército numeroso y bien pertrechado de armas puede ser derrotado por uno más pequeño y menos armado pero que está galvanizado por la conciencia de luchar por una causa justa y por una voluntad reforzada por vínculos de solidaridad y por un sentimiento de pertenencia y amor a una sociedad integrada, a una patria. En la vida política, un partido unido es capaz de suscitar más fuertes adhesiones y lograr sus objetivos mejor y más eficazmente que otro en el que existe división y conflictos internos (Razeto, 2011a).

En este momento estamos en la búsqueda de nuevos conceptos de economía, y gracias a Razeto y sus teorías, se han desplegado procesos históricos, luchas sociales, experiencias organizativas, dinámicas ideológicas y políticas de formidable magnitud. Tales procesos han seguido dos direcciones principales: la de una transformación "sistemática" o macrosocial, por un lado, en la que el énfasis ha sido puesto en la acción política y en proyectos de economía estatal o socialista, y la de creación de alternativas microeconómicas, por el otro, en las que se enfatiza la experimentación y el desarrollo de iniciativas sociales, cooperativas autogestionarias, comunitarias y solidarias; los resultados reales de estos esfuerzos, luchas y trabajos son satisfactorios en la medida en que se puedan demostrar a partir de la investigación y cómo se avanza en el proceso.

Para comprender las causas y tratar de superar la insuficiencia existente en los conceptos de comunidad, solidaridad, cooperativismo y asociatividad, entre otros, se ha estudiado e investigado la Teoría Económica Comprensiva del profesor Razeto, proporcionando así el conocimiento para un desarrollo del sentido profundo que tiene como trasfondo la realización, hasta el momento, de la investigación y caracterización del Factor C-comunidad (Valencia, 2012).

El Factor C-comunidad es el resultado del trabajo en compañerismo, en amistad, como fruto de la autogestión, la ayuda mutua, la cooperación y la solidaridad recíproca, que hacen de la empresa de economía solidaria una autentica comunidad, lo cual desarrolla un elevado sentido de pertenencia de sus integrantes hacia esta. El Factor C-comunidad da la clave para comprender la lógica con que operan las empresas de economía solidaria, porque incorpora la solidaridad activa al proceso de producción, generando una particular productividad que condiciona y determina los demás factores. Sin embargo, lo que caracteriza una empresa de economía solidaria es la existencia de un Factor C-comunidad que sea el intensivo, el estructurante central de la empresa solidaria, el articulador y potencializador de los demás factores económicos (Arango, 2005).

Entonces, a través de procesos históricos determinados y de investigaciones anteriores realizadas a la luz de la teoría razetiana, se han identificado seis principales factores, a saber:

- Factor financiamiento. Normalmente está constituido por una cierta cantidad de dinero o por alguna capacidad de crédito que permite contratar factores y establecer relaciones económicas en el mercado. La forma que este factor asume normalmente es el dinero; pero puede tratarse de algún otro medio con el cual se logra convocar, atraer y adquirir los recursos y factores necesarios.

- Factor laboral o fuerza de trabajo. Son las capacidades físicas e intelectuales de cada persona, que la hacen apta para ejecutar una serie de actividades laborales participando directamente en el proceso económico.

- Factor gestión. Es decir, un sistema unificado de coordinación y dirección de las funciones y 
actividades económicas; un mecanismo de toma de decisiones por medio del cual cada empresa responde oportunamente a las cambiantes condiciones en que opera y coordina eficazmente la actividad de todos sus componentes. Incluye el elemento de poder (línea de mando) y el aparato administrativo.

- Factor medios materiales o físicos. Es el conjunto de los elementos físicos, las instalaciones, los instrumentos y equipamientos, los insumos y las materias primas, necesarios para efectuar concretamente el proceso técnico de trabajo, en la producción y en las demás funciones económicas.

- Factor tecnología. Es el conjunto de conocimientos, saberes e información relativos a unos procesos y sistemas técnicos de producción, organización del trabajo y comercialización. Es un "saber hacer" valorizado como tal. Es un factor que se desarrolla por medio del estudio, se perfecciona con un conocimiento práctico que se acumula en cada persona, empresa o sociedad, y que se va cristalizando y sedimentando en instrumentos de trabajo, en sistemas técnicos, en rutinas de trabajo o en formas de hacer las cosas.

- Factor C-comunidad. Es un nuevo factor que integra los modelos y análisis económicos, junto con el trabajo, el capital y la tecnología, entre otros. Si en cualquier empresa, hasta en las más grandes y modernas, se pusiera o incrementara la solidaridad, con seguridad serían más productivas y eficientes. $\mathrm{Si}$ en el mercado hubiera más solidaridad, el mercado sería más perfecto y funcionaría de mejor manera. Si en las políticas públicas y en las decisiones económicas del Estado hubiera mayores dosis de solidaridad, esas políticas serían mejores y sus resultados más eficientes. Esa es la fuerza del "Factor C”. Simplemente porque con la letra c comienzan muchas palabras que lo identifican: compañerismo, comunidad, cooperación, colaboración, comunión, coordinación y otras (Razeto, 1994b).

\section{Factores económicos productivos}

En la figura 3 se representa la integración de los factores económicos productivos, describiéndolos y haciendo un reconocimiento complejo a las características que los diferencian, pero que a su vez conllevan a su unificación, donde es fácil comprender que no hay una actividad económica si no no existe cooperación en el trabajo, organización en la gestión, racionalidad en el uso de los materiales y en el elemento económico, un uso colaborativo de conocimientos y la unificación entre las personas y su comunidad.

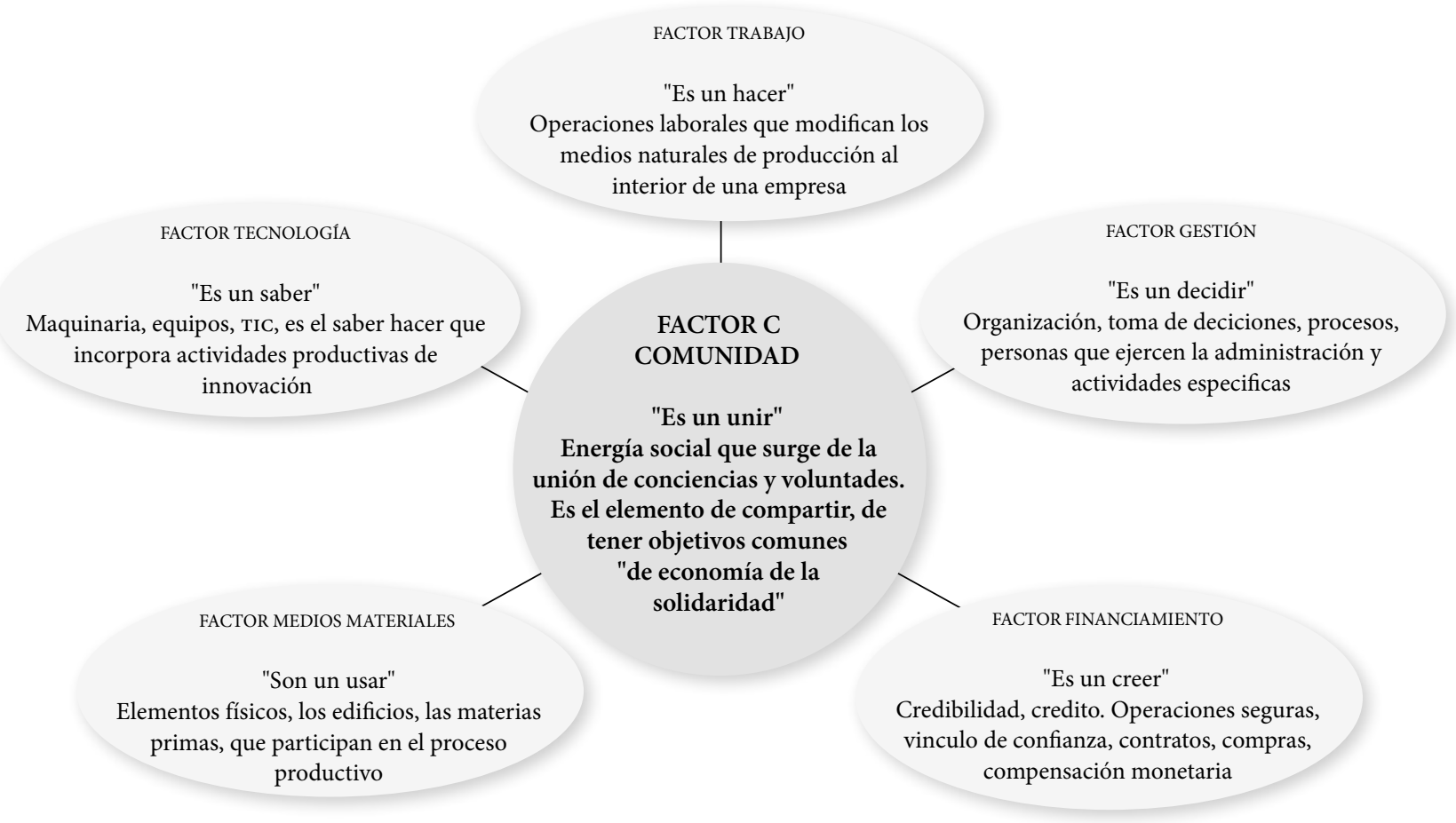

Figura 3. Integración de los factores

Fuente: adaptado por los autores, a partir de Luis Razeto 


\section{Metodología}

Tabla 3. Estructura general de la investigación

\begin{tabular}{|c|c|c|c|}
\hline Iniciación & Trabajo de campo & Análisis e interpretación & Socialización de resultados \\
\hline $\begin{array}{l}\text { - Ajustes teóricos y } \\
\text { metodológicos al proyecto } \\
\text { - Revisión y análisis de fuentes } \\
\text { primarias secundarias }\end{array}$ & $\begin{array}{l}\text { - Diseño de instrumentos } \\
\text { - Elección a criterio de } \\
\text { organizaciones y personas } \\
\text { - prueba piloto de instrumentos } \\
\text { - revisión documentos fuente de } \\
\text { las organizaciones }\end{array}$ & $\begin{array}{l}\text { - Definición de categorías desde } \\
\text { lo cualitativo } \\
\text { - Definición de categorías desde } \\
\text { lo cuantitativo } \\
\text { - Definición de categorías } \\
\text { emergentes } \\
\text { - Análisis e interpretación de los } \\
\text { resultados }\end{array}$ & $\begin{array}{l}\text { - Generacion de nuevos } \\
\text { conocimientos } \\
\text { - Ponencias nacionales e } \\
\text { internacionales } \\
\text { - Foros de participación }\end{array}$ \\
\hline
\end{tabular}

Fuente: elaboración propia

La metodología se basó en un estudio cualitativo de tipo etnográfico, con la utilización de diversas técnicas provenientes tanto del enfoque cualitativo como del cuantitativo, que permitió caracterizar el Factor C-comunidad en las asociaciones y cooperativas de pequeños y medianos productores agropecuarios de las zonas Sinifaná y San Juan, en el suroeste antioqueño. A su vez tuvo un cronograma de desarrollo basado en iniciación del proyecto, trabajo de campo, análisis e interpretación y socialización de resultados (tabla 3).

La investigación cualitativa de tipo etnográfico propone ayudar a interpretar el entorno a partir de un análisis, en el que se observa lo que dicen, hacen, actúan y piensan sus actores y protagonistas, y que a su vez posee un rigor teórico, técnico y metodológico con principio y flexibilidad para el registro y análisis de la información.

Etimológicamente, el término etnografía significa la descripción Grafé del estilo de vida de un grupo de personas habituadas a vivir juntas Ethnos (Rueda, 2007). Por tanto, el Ethnos, que en este caso es la unidad de análisis, fue el grupo humano que constituye las entidades $u$ organizaciones pequeñas y medianas asociativas y agropecuarias, cuyas relaciones están reguladas por la asociatividad y por costumbres, derechos y obligaciones recíprocos. En la economía solidaria las pequeñas y medianas asociaciones son unidades sociales que pueden ser estudiadas etnográficamente.

La metodología etnográfica, como un método abierto de investigación en terrenos en los que caben las encuestas, las técnicas no directivas fundamentalmente, la observación participante, las entrevistas no dirigidas y la residencia prolongada con los sujetos de estudio, es el conjunto de actividades que se suele designar como trabajo de campo, y cuyo resultado se emplea como evidencia. Los fundamentos y las características de esta flexibilidad o apertura radican, precisamente, en que son los actores, y no el investigador, los privilegiados para expresar en palabras y en prácticas el sentido de su vida, su cotidianidad, sus hechos extraordinarios y su devenir. Este estatus de privilegio replantea la centralidad del investigador como sujeto asertivo de un conocimiento preexistente, convirtiéndolo, más bien, en un sujeto cognoscente que deberá recorrer el arduo camino del des-conocimiento al re-conocimiento.

Este proceso tiene dos aspectos: en primer lugar el investigador parte de una ignorancia metodológica y se aproxima a la realidad que estudia para conocerla; esto es: el investigador construye su conocimiento a partir de una supuesta y premeditada ignorancia. Cuanto más sepa que no sabe, o cuanto más ponga en cuestión sus certezas, más dispuesto estará a aprender la realidad en términos que no sean los propios. En segundo lugar, el investigador se propone interpretar-describir la entrevista no dirigida y la observación participante; el instrumento es el mismo investigador con sus atributos socioculturalmente considerados en una relación social de campo (Guber, 2001). La tradición etnográfica y los métodos cualitativos constituyen una decisiva ayuda para un mayor entendimiento crítico de las situaciones y fenómenos educativos $\mathrm{y}$, por consiguiente, para una más adecuada y consciente intervención (Torres, 1998).

Sin embargo, en la investigación se utilizó como técnicas la entrevista semiestructurada para gerentes, líderes y personas clave en la organización. Se hizo revisión documental y registro en fichas de contenido y de análisis. Los instrumentos se validaron por expertos de la academia y de organizaciones consideradas en el estudio.

Con esta metodología se logró establecer y conocer aspectos importantes en las organizaciones objeto de la investigación, en las que se evidenció que se 
integran en una asociación a partir de la necesidad de innovar en algún producto que cosechan, con la esperanza de ingresar en un mercado específico y obtener así las ganancias que les permitan mejorar la calidad de vida de los asociados:

[...] La organización surgió por la necesidad de mejorar la calidad del producto, específicamente en la calidad del lavado porque vieron un vacío que era común entre todos, al mejoramiento del medio ambiente y al bienestar de la familia y de las personas que trabajan allí. La idea surgió de la comunidad [...] Había ya mucha gente trabajando en cafés especiales y apuntándole mucho al medio ambiente y a la calidad del café, y de hecho al mejoramiento y al bienestar de la familia, de las personas que allí trabajan, a eso apuntan estos grupos, las comercializadoras de café como Indicafe, Reiforez, Expreso, una de las cosas que piden ellos es eso, que se conserve el medio ambiente y que no se le vulneren los derechos al trabajador, entonces aquí surgió ese grupo pero a alguien en la vereda se le ocurrió decir una vez oiga y nosotros porque no hacemos un beneficiadero comunitario, nosotros, el grupo, porque la idea, como les parece a ustedes que a pesar de que Jardín por el ecosistema muy favorable produce un café de mucha calidad, el problema era en la calidad por el lavado, entonces alguien dijo hagamos un beneficiadero comunitario porque a nosotros en nuestros beneficiaderos nos queda muy difícil lavar café todos los días, por muchas circunstancias y el café pierde su sabor en el tiempo, si usted se demora 3 o 4 días ya se vuelve amargo o de sabor áspero, ellos tienen varias denominaciones, entonces alguien dijo, hagamos el beneficiadero nosotros y de ahí surgió la idea [...]

Surge asimismo de las entrevistas semiestructuradas la evidencia de una serie de dificultades que deben sobrellevar los productores agropecuarios para salir adelante con los proyectos asociativos que buscan además ser sustentables y sostenibles, pero que prueban asimismo el Factor C-comunidad:

[...] uno tiene que tener es como mucha voluntad $i$ cierto? porque esto no es para uno estar diciendo de un día para otro que lo va a lograr, esto tiene uno que insistir mucho. Nosotros llevábamos por ahí 8 o 10 años en este proyecto, nos costó mucho y se vino a lograr ya en este año $[\ldots]$

Se identificó que al acudir en ayuda de las entidades del gobierno como alcaldías, gobernaciones y otras entidades como las Corporaciones Autónomas
Regionales (CAR), se logra alcanzar ayudas de tipo económico, material, tecnológico y trabajo, que son factores claramente expuestos por el profesor Razeto en su teoría y que convergen el factor integrador C-comunidad en el logro de los objetivos asociativos; sin embargo, no se ejecutaban en su totalidad, haciéndolos vulnerables hacia otras ayudas y proyectos:

[...] La asociación comenzó con la vinculación de uno de los asociados a la Umata y luego a un programa de la gobernación "alianzas productivas" con el tema productivo de las abejas [...] Lo único que sobrevivió fueron las abejas, a cada junta de acción comunal, a tres socios les regalaron tres colmenas y de ahí pues cada asociado decidimos crecer las colmenas y así aumentamos. Después de muchos intentos logramos conformar Asoapibe en el 2000, el proyecto fue en el 94.

Al desarrollar la investigación, se buscó generar un gran aporte a la sociedad acerca de esta nueva teoría existente y conocida por pocos, que se pudo evidenciar en las asociaciones y cooperativas de pequeños y medianos productores agropecuarios del suroeste antioqueño, que son fuente de desarrollo social y económico a nivel departamental y nacional.

La población objeto de estudio fueron los pequeños y medianos productores agrícolas que conforman las asociaciones y cooperativas, seleccionadas a criterio, y establecidas en áreas urbana y rural de las zonas Sinifaná y San Juan, del suroeste antioqueño, de acuerdo con registros del 2009 de la Cámara de Comercio y la Gobernación de Antioquia. Las organizaciones se seleccionaron de acuerdo con la existencia real,

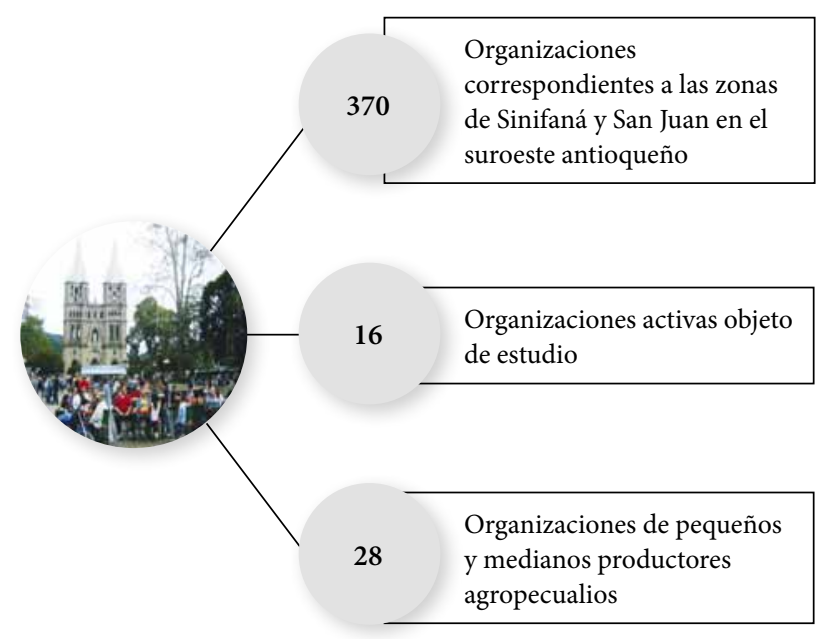

Figura 4. Registro de asociaciones del suroeste antioqueño Fuente: Cámara de Comercio de Antioquia, 2010 
Tabla 4. Registro de asociaciones del suroeste antioqueño

\begin{tabular}{|l|c|}
\hline \multicolumn{1}{|c|}{ Tipo } & Número de organizaciones \\
\hline Organizaciones registradas en la Cámara de Comercio. & 10.962 \\
\hline Organizaciones correspondientes a las zonas de Sinifaná y San Juan, en el suroeste antioqueño. & 370 \\
\hline Organizaciones de pequeños y medianos productores agropecuarios. & 28 \\
\hline Organizaciones activas objeto de estudio, & 16 \\
\hline
\end{tabular}

Fuente: Cámara de Comercio de Antioquia, 2010

la vigencia de la actividad económica, el rol institucional y el liderazgo para dinamizar su organización en el entorno municipal, subregional y departamental. Asimismo, se tuvo en cuenta que estuvieran activas en su conformación y dinamismo productivo y que cumplieran con la característica de ser pequeñas y medianas producciones agropecuarias (figura 4).

Para abordar el estudio se tuvieron en cuenta las fuentes primarias y secundarias provenientes de los informantes clave, los archivos institucionales, las cámaras de comercio, la planeación departamental y municipal, entre otros.

Según la Cámara de Comercio, en el departamento de Antioquia se encontraron inscritas 10.962 organizaciones del sector de solidario, distribuidas como se muestra en la tabla 4 y la figura 4.

\section{Resultados}

Por las características metodológicas que se trabajaron en la investigación, estudio cualitativo de tipo etnográfico, con herramientas cualitativas y cuantitativas, en las que se aplicaron algunos de los componentes que lleva inmersa esta estructura, se presentan los resultados obtenidos, así: la herramienta de tipo cuantitativo estuvo representada en una encuesta cuyo universo muestral estuvo apoyado en:

$$
n=\frac{N z_{\alpha l 2}^{2} P(1-P)}{(N-1) e^{2}+z_{\alpha l 2}^{2} P(1-P)}
$$

Donde:

$\mathrm{N}=$ total asociados productores pertenecientes a las organizaciones de pequeños y medianos productores agropecuarios de las zonas de Sinifaná y San Juan, del suroeste antioqueño.

$$
\begin{aligned}
& \mathrm{a}=\text { nivel de confianza }=0,5 \\
& \mathrm{P}=\text { proporción }=0,5 \\
& (1-\mathrm{P})=\text { complemento de } " \mathrm{P} "=1,95 \\
& \mathrm{E}=\text { error estándar }=0,05
\end{aligned}
$$

El ejercicio arrojó una muestra de 121 personas, de las cuales 99 son de sexo masculino y 22 de sexo femenino (figura 5). Se encuentran en un rango de edad entre 21 y 80 años (figura 6).

Cabe resaltar que son los hombres entre 42 y 52 años de edad son el número más representativo en estas organizaciones agropecuarias, situación que es comprensible pues son ellos quienes ejercen la mayoría de las actividades propias del campo con relación a las mujeres, en el sector rural, que en su mayoría están dedicadas a las labores del hogar y al cuidado de los hijos. Al unir la técnica cuantitativa con la variable cualitativa de la entrevista no dirigida, se observó que las mujeres, aunque ejercen una influencia alta en la sostenibilidad económica de los hogares de la región, por ser la base en el cuidado de los miembros de la familia, no participan activa y frecuentemente en las organizaciones solidarias.

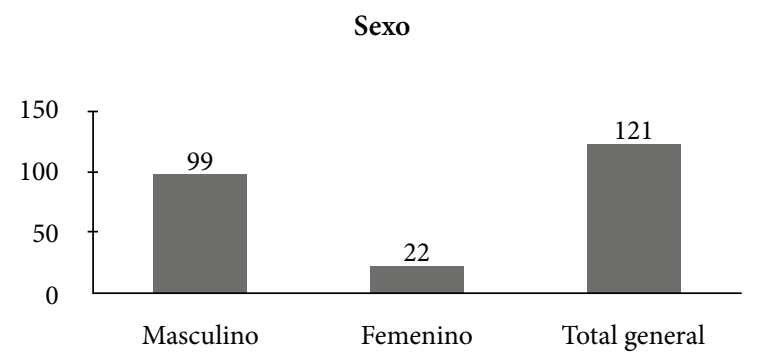

Figura 5. Sexo de la población investigada Fuente: elaboración propia

\section{Edad}

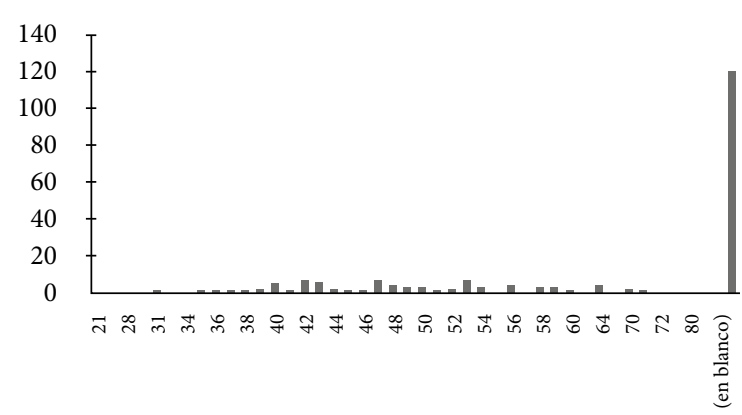

Figura 6. Nivel de educación de la población estudiada Fuente: elaboración propia 
Cuando se habla de mejoramiento de la calidad de vida, situación que se pretende lograr con las actividades propias de la asociatividad y la relación que tiene el desarrollo del Factor C-comunidad en ellas, la pretensión del ser humano es suplir necesidades básicas alrededor de la educación, la salud, y la vivienda. Las figuras 5 a la 10 nos muestran la caracterización etnográficas que arrojó el componente cualitativo de la investigación.

En el caso estudiado, el nivel máximo de educación alcanzado es técnico, en su mayoría en formación para el trabajo agropecuario; sin embargo, la mayoría de los encuestados sólo ha estudiado la primaria, muchos sin haberla terminado (figura 7).

De las 121 personas encuestadas, el 93\% son asociados directos de las organizaciones; asimismo, el 86\% manifiesta haber mejorado su calidad de vida, al obtener vivienda propia, viviendas que se encuentran ubicadas en su mayoría en estrato 2 (figura 8).

Al desarrollar la entrevista semiestructurada, propia del estudio cualitativo, los resultados obtenidos evidenciaron un fuerte contraste entre las características propias del ofrecimiento de mejoramiento de calidad de vida en servicios y beneficios y la propuesta de la teoría razetiana en relación con el Factor C-comunidad y sus objetivos.

La investigación permitió caracterizar e identificar el Factor C-comunidad en las poblaciones objeto de la investigación a través de las experiencias analizadas y vivenciadas por los miembros de las comunidades.

Se identificó el Factor C-comunidad en las asociaciones y cooperativas de pequeños productores agropecuarios de las zonas de Sinifaná y San Juan objeto de la investigación, como tratar de compartir el conocimiento y la gestión; el trabajo conjunto; ayudar a los demás; asociarse para mejorar la calidad de vida; trabajar en equipo con dedicación y esfuerzo para obtener buenos resultados en los proyectos que se proponen realizar; cada habitante de la comunidad aporta todo lo que puede, ya sea de tipo económico, material o con trabajo o en la idea que desean ejecutar. Las ganas de salir adelante motiva a los integrantes de las asociaciones y cooperativas para que día a día se generen más ideas y se consolide una gestión sustentable y sostenible en el tiempo:

[...] A mí me sigue motivando es la calidad humana, yo creo que cuando pensamos en el ser humano y en mejorar su vida, sus capacidades, en este caso económicas, yo creo que vamos a mejorar mucho, y vamos a quedar
Nivel máximo de educación alcanzado

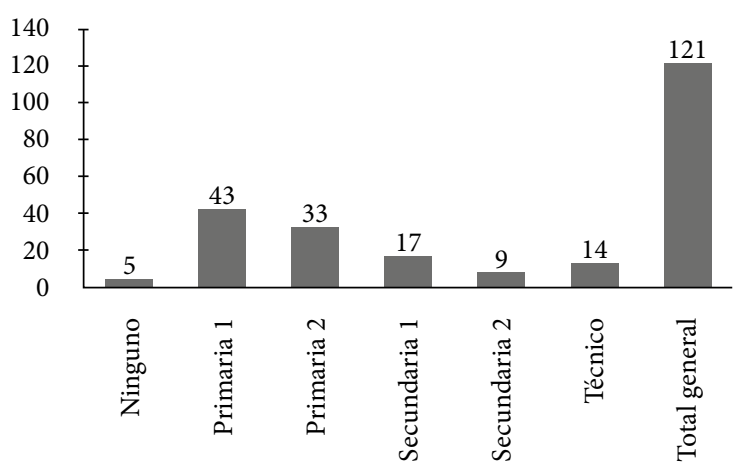

Figura 7. Afiliación a la asociación

Fuente: elaboración propia

Filiación

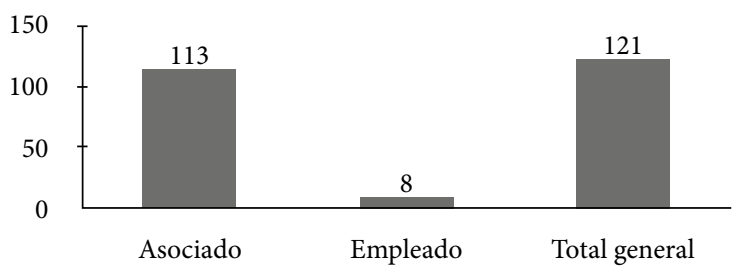

Figura 8. Tenencia de la vivienda en los entrevistados Fuente: elaboración propia a partir de la recolección de datos

con una sociedad mejor, que esa aspiración más grande puede ser que yo en 8 o 10 años, dos o tres años pueda decir: no, es que la gente que está a mi lado se está ganando un salario mínimo [...]

En las asociaciones y cooperativas de pequeños productores agropecuarios de las zonas de Sinifaná y San Juan, se evidenció que al pedir ayuda a las entidades del gobierno como alcaldías, gobernaciones y otras entidades públicas, logran recibir ayudas de diferente tipo, pero que el interés, la unión de voluntades y la cooperación entre ellos y los miembros de la comunidad, es lo que los incita a permanecer frente a las dificultades:

[...] La organización comenzó hace 2 años más o menos. Empecé a convocar a algunos y la idea buena, muy rico y hagámosle y logramos tener 48 asociados y en la actualidad somos 38 , siempre han desistido $10 \mathrm{y}$ espero que todavía salgan unos, que uno no los ve como tan compatibles ni tan fuertes o al menos con tanto respeto a la Asociación, porque es que esto es como una familia y por tanto se debe manejar de una manera muy buena, 
pero yo siempre les dije a ellos, vea de aquí a nadie vamos a echar, yo creo que de aquí la gente se va a ir echando sola, tenemos un reglamento muy bueno, muy amplio el cual nos da mucha libertad pero también nos aprieta en el momento en que nos debe apretar, la fortaleza grande, grande de la Asociación es el deseo de la gente de salir adelante. [...] La asociación tuvo una crisis los asociados desertaron, sin embargo la asociación jurídicamente no desapareció, luego volvió a resurgir con 65 personas interesadas en el tema y se cambió el objeto social, el nombre y los estatutos.

La dinámica del Factor C-comunidad está presente en los habitantes de la región que desean mejorar la calidad de vida de sus familias, de tal manera que sus

Propiedad de la vivienda

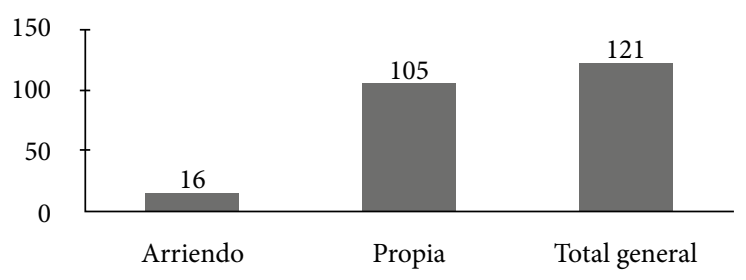

Figura 9. Pertenencia o participación en el uso de las viviendas de los entrevistados

Fuente: elaboración propia

Estrato de vivienda

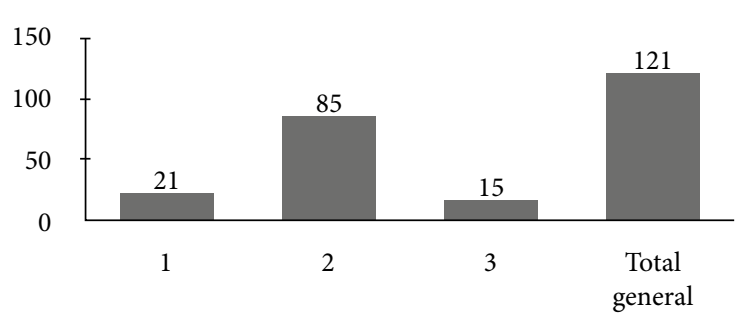

Figura 10. Estratificación de las viviendas de los entrevistados Fuente: elaboración propia

hijos desde muy pequeños muestren interés y ganas de trabajar por la comunidad y que continúen con la tradición de sus padres sin desplazarse a la ciudad en busca de un futuro mejor por falta de oportunidades en su tierra:

[...] Ahí nació la idea y el deseo de ver un grupo, como yo les decía a ellos vea, tranquilamente con mis conocimientos yo podría irme e iniciar un proceso solo y llegar a llenarme como dice el cuento y llegar a llenar mi forma de vida, pero la idea no era esa, la idea era iniciarlo y decir cómo les decía ahorita, de mi nació la idea de esto y en este momento se están beneficiando tantas personas, o sea sentir el orgullo de poder haber ayudado a varios personas [...] El gusto por la administración y no dejar perder lo que ya estaba construido [...] Porque a mí siempre me han gustado las empresas porque yo en el SENA hice un curso de empresarismo y fue la Administración la que me cogió cortico porque este proyecto lo iban a dejar perder, hay mucha gente para este proyecto pero se fueron retirando entonces para no dejarlo perder nos cogieron a unos poquitos, nos cogió el secretario de Gobierno en ese momento [...] Hermano venga que usted me sirve como socio para una truchera; ¿qué tengo que hacer? Ah no, fotocopia de la cédula, y vamos y firmamos, entonces yo le dije, bueno en dónde es el terreno, es en tal parte, entonces nos vinimos a mirar el terreno, a quiénes son los socios, fulano y fulano, recójalos pues que vamos a empezar que a mí me gusta hacer las cosas ligerito, conocemos el terreno y empezamos a trabajar [...]

La definición del concepto de Factor C-comunidad afirmado en la región objeto de estudio, se expresa como la realización de la autogestión en las organizaciones; como el compartir objetivos comunes; como la colaboración para obtener el mejoramiento de todos; como la unión de conciencias, sentimientos y voluntades. Se encontró que efectivamente es el factor integrador y organizador que se encuentra inmerso en las actividades que desarrollan los productores agropecuarios.

\section{Conclusiones}

\section{Como lo expresa Luis Razeto:}

es Factor C, simplemente porque con la letra c comienzan muchas palabras que lo identifican: compañerismo, comunidad, cooperación, colaboración, comunión, coordinación y otras. Hace años, en Venezuela, yo explicaba el "Factor C" en una comunidad de trabajo, y un hombre ya anciano y de larga trayectoria de acción social me dijo: "A ese Factor $C$ yo le pondría una $C$ mayúscula, porque para mí es el factor Cristo". Y así quedó, con mayúscula (1994b).

La realización de la investigación identificó la dinámica del Factor $\mathrm{C}$-comunidad como el principal motivante de una comunidad para lograr desarrollar exitosamente un proyecto o idea; asimismo, que existe un claro liderazgo de una o varias personas dentro de las organizaciones, persona que motiva y transmite sus ideas a los demás y que genera la sinergia de la 
cooperación entre todos los miembros, evidenciando con claros ejemplos el éxito que se logra al trabajar todos en unión, en común unidad. También es claro que los proyectos que han fracasado ha sido por no reconocer que se necesita algo más que dinero y medios materiales para lograr los objetivos.

Se equipara la forma de operar del Factor C-comunidad por sus características propias, a su vez se brindó conocimiento a las comunidades en qué consiste la teoría de este factor debido a que son pocos los que lo identifican desde se naturaleza; se cuenta con muy poco conocimiento por parte de la sociedad debido a que son pocas las teorías existentes que analizan lo importante de trabajar en comunidad.

Por ello se compartió con las comunidades la definición del Factor C-comunidad, lo que les permitió reconocer e identificar su importancia. A su vez, se propuso a las instituciones participantes en la investigación elementos de capacitación en el conocimiento de la normatividad y reglamentación en la conformación de organizaciones del sector solidario, como también el curso básico que promueven las organizaciones del Estado.

La investigación permitió caracterizar y aportar comprensión a los problemas actuales relacionados en el contexto regional, en tanto que abordó un tema de interés como es la economía solidaria y su búsqueda de mejor bienestar para los grupos poblacionales que intentan resolver su situación de manera autónoma y autogestionaria; problemas que serían resueltos ofreciendo formación, capacitación y educación en todo el planteamiento solidario, de asociatividad y emprendimiento, lo que a su vez permitirá ejecutar debidamente los procesos organizacionales y de desarrollo de proyectos.

Se identificó que cuando se trabaja en equipo y se tienen buenas ideas asociativas alrededor de desarrollos productivos por parte de las personas que integran una comunidad, se logra obtener con mayor facilidad los recursos que son brindados por el Gobierno y los entes estatales. De igual manera, las oportunidades para acceder a los recursos económicos se obtienen fácilmente si los servicios y beneficios que generen las organizaciones asociativas se distribuyen entre sus miembros, incrementando con ello la participación en el bienestar social.

\section{Referencias}

Antioquia. Gobernación de Antioquia. (2004). Gobernación de Antioquia. Recuperado de http://antioquia.media. vcb.com.co/antioquia.gov.co/antioquia-v1/organismos/planeacion/descargas/instructivos/amaga.pdf

Arango, M. (2005). Manual de cooperativismo y economia solidaria. Medellin: Teoría del Color.

Guber, R. (2001). La etnografia, metodo, campo y reflexibilidad. Bogotá: Norma.

Prieto, R. y Galas, C. (2006). Economia solidaria de la obsesión por el lucro a la redistribución con equidad. Barcelona: Icaria Editorial S.A.

Razeto, L. (1994a). Fundamentos de una teoría económica comprensiva. Santiago de Chile: PET.

Razeto, L. (1994b). Economia de solidaridad y mercado democratico: fundamentos de una teoría económica comprensiva. Santiago de Chile: Ediciones Uvirtual.

Razeto, L. (2011). Luis Razeto.net. Recuperado de http:// www.luisrazeto.net/content/xv-la-productividad-oculta-y-la-productividad-manifiesta-del-factor-c

Razeto, L. (2011). Nueva civilización.NEt. Recuperado de http://www.luisrazeto.net/content/xv-la-productividad-oculta-y-la-productividad-manifiesta-del-factor-c

Rueda Beltran, M. (2007). Revista Mexicana de Investigacion Educativa. Julio-Septiembre. Recuperado de http:// www.comie.org.mx/v1/revista/visualizador.php?artic ulo=ART34009\&criterio $=$ http://www.comie.org.mx/ documentos/rmie/v12/n034/pdf/N34I.pdf

Torres Santomé, J. (1998). La investigación etnografica y la reconstrución critica en educación. Madrid: Ediciones Morata S.A.

Valencia, L. M. (2012). Factor comunidad en las asociaciones y cooperativas de pequeños y medianos productores agropecuarios en las zonas del Sinifaná y San Juan en el suroeste antioqueño. (L. A. Gallego, entrevistador). 